\title{
Microstructures in All-Inkjet Printed Textile Capacitors with Bilayer Interfaces of Polymer Dielectrics and Metal-Organic Decomposition Silver Electrodes
}

\author{
Inhwan Kim, Beomjun Ju, Ying Zhou, Braden M. Li, Jesse S. Jur* \\ Fiber and Polymer Science Program, North Carolina State University \\ *Corresponding author: jsjur@ncsu.edu
}

\section{Supporting Information}

Table S-1. Sample fabrication conditions

Page 2

Figure S-1. Rheology of the inkjet-printed inks

Page 2

Figure S-2. Surface tension of inkjet-printed inks

Page 3

$\begin{array}{ll}\text { Figure S-3. Water contact angle on film and textile substrates } & \text { Page } 3\end{array}$

Figure S-4. Optical image of the pristine PET woven textiles $\quad$ Page 3

Figure S-5. Cross-sectional SEM images at 500x for film and textile capacitors $\quad$ Page 4

Figure S-6. Electromechanical performance evaluation setup in Figure $7 \quad$ Page 5

Figure S-7. Relative capacitance change in film and textile capacitors $\quad$ Page 6

Figure S-8. Absolute capacitance change of the capacitors (combined plot) $\quad$ Page 7 
Table S-1. Sample fabrication conditions based on factorial experimental design.

\begin{tabular}{lcc|c|c}
\hline & & \multicolumn{3}{c}{ No. of PVP/PMF Print Process(es) } \\
\cline { 2 - 5 } & 1 & Film or Textile 1-1 & Film or Textile 1-2 & Film or Textile 1-3 \\
\cline { 2 - 5 } & 2 & Film or Textile 2-1 & Film or Textile 2-2 & Film or Textile 2-3 \\
$\begin{array}{c}\text { No. of UA } \\
\text { Print } \\
\begin{array}{c}\text { Process(es) } \\
$\cline { 2 - 4 }\end{array}\end{array} & 3 & Film or Textile 3-1 & Film or Textile 3-2 & Film or Textile 3-3 \\
\hline
\end{tabular}

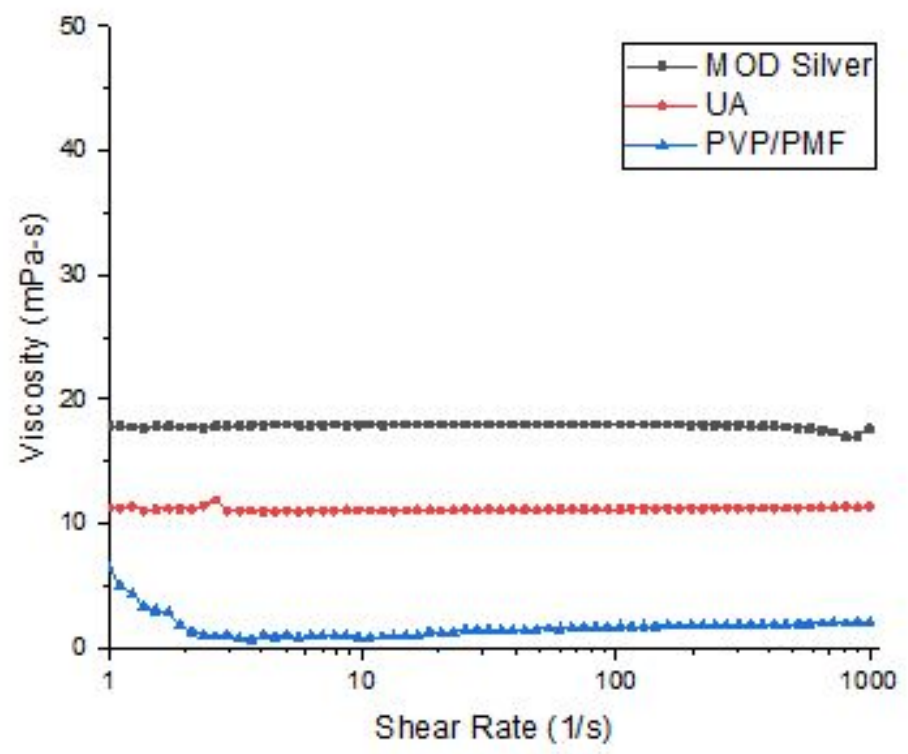

Figure S-1. Rheology of the inkjet-printed inks. 


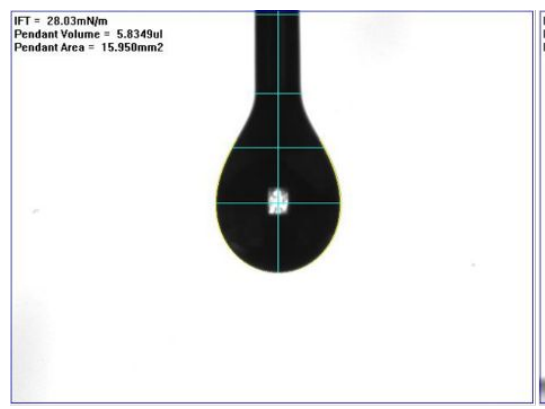

MOD Silver Ink Surface tension: $28.03 \mathrm{dyn} / \mathrm{cm}$

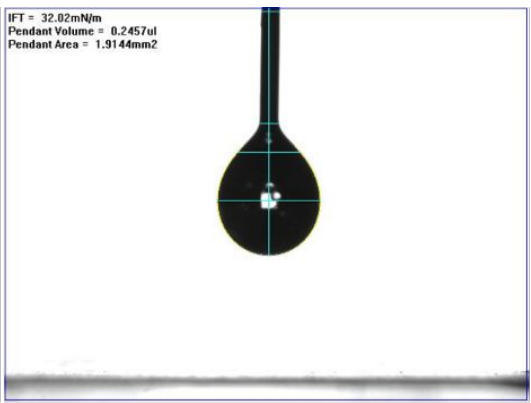

Urethane-acrylate ink Surface tension: $\mathbf{3 2 . 0 2} \mathrm{dyn} / \mathrm{cm}$

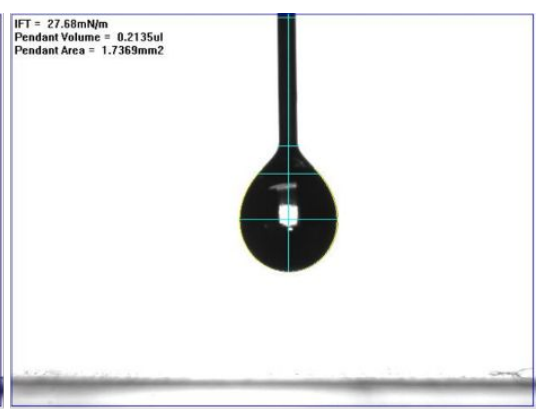

PVP/PMF ink Surface tension: $27.68 \mathrm{dyn} / \mathrm{cm}$

Figure S-2. Surface tension of inkjet-printed inks.

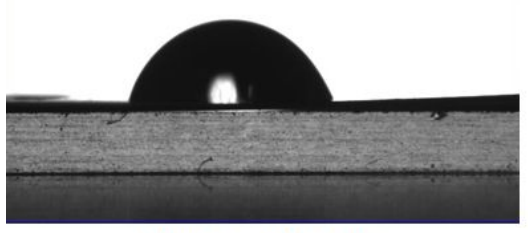

Water on PET Film Contact angle: $69.5^{\circ}$

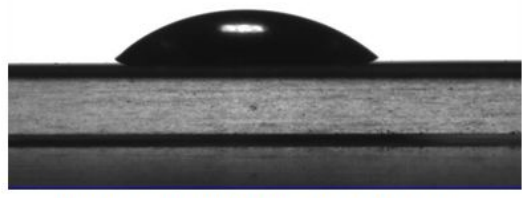

Water on UVO Treated PET Film Contact angle: $40.3^{\circ}$

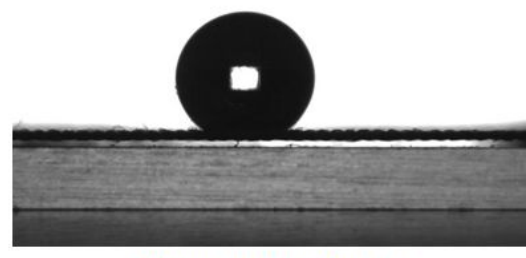

Water on PET Textile Contact angle: $129.6^{\circ}$

Figure S-3. Water contact angle on film and textile substrates.
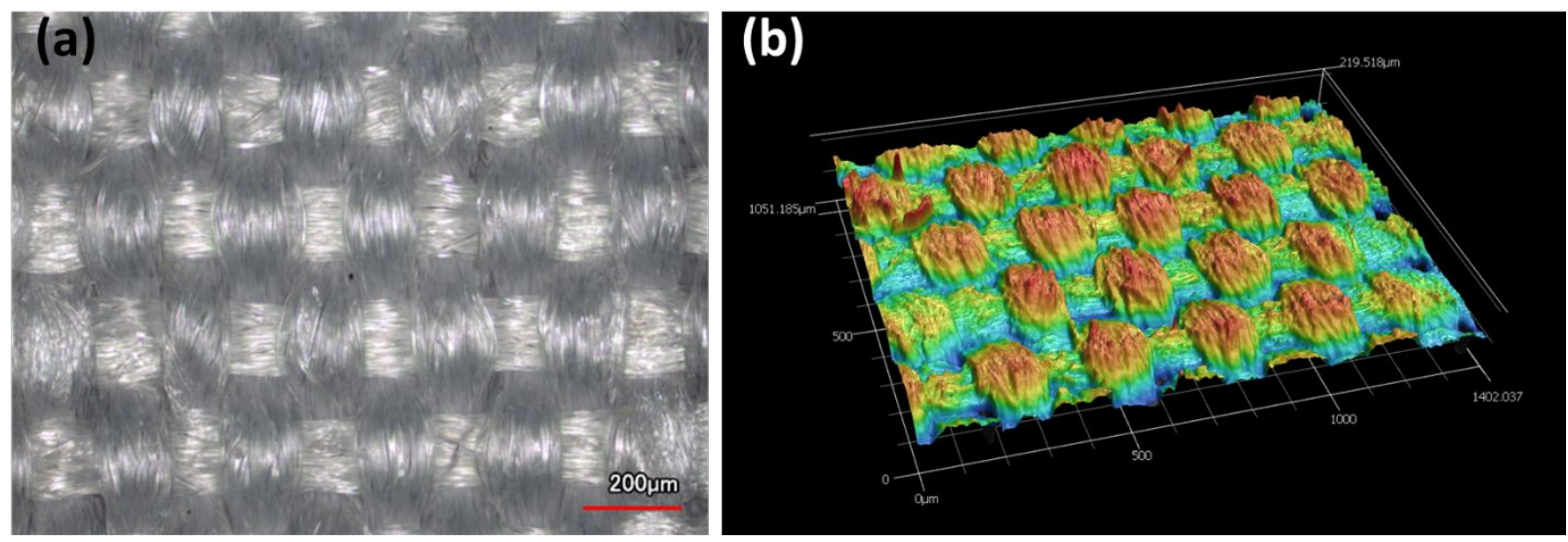

Figure S-4. Optical image of the pristine PET woven textiles (a) optical image (10x), and (b) 3D topography obtained from laser confocal scanning. 

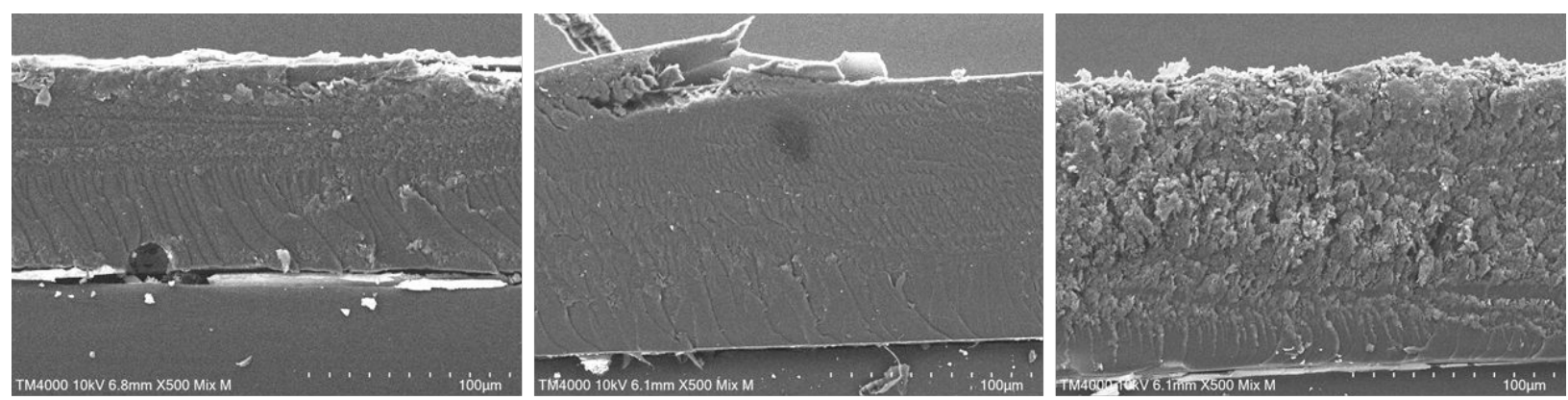

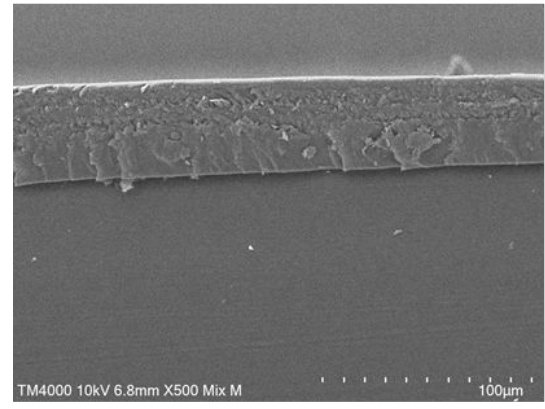

Film 2-2

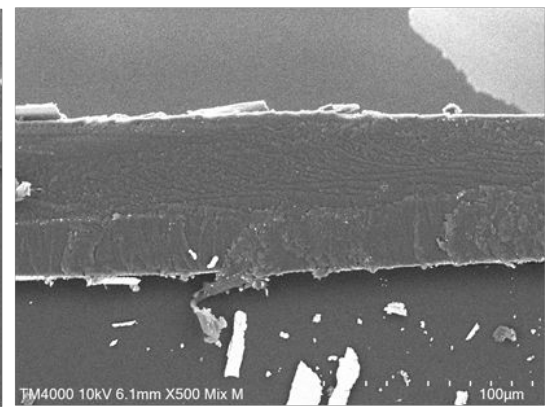

Film 3-1

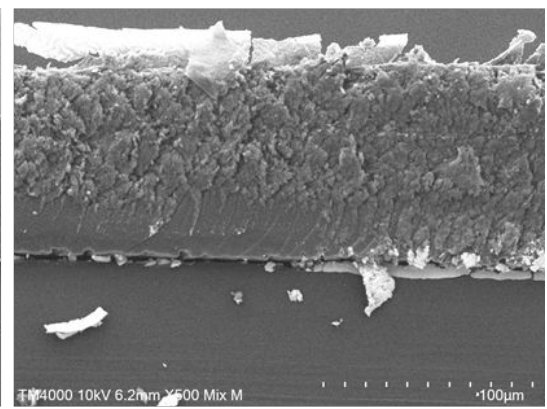

Film 3-3
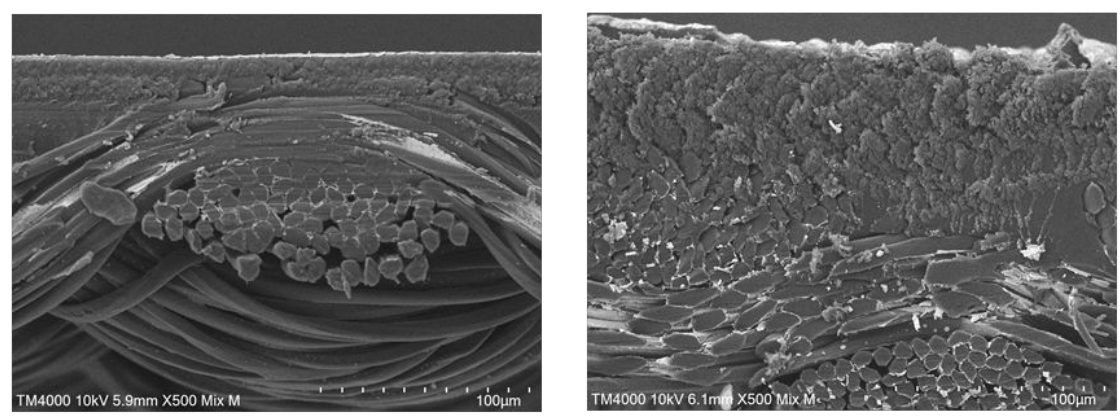

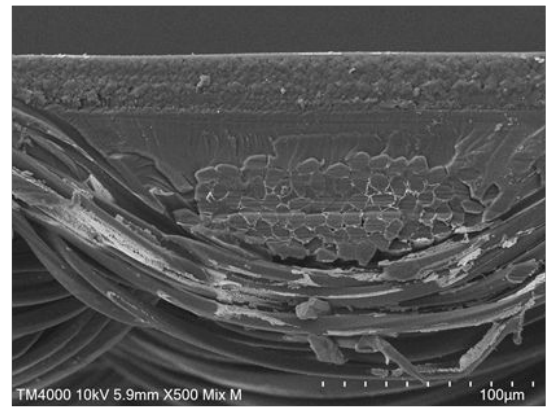

Textile 3-1

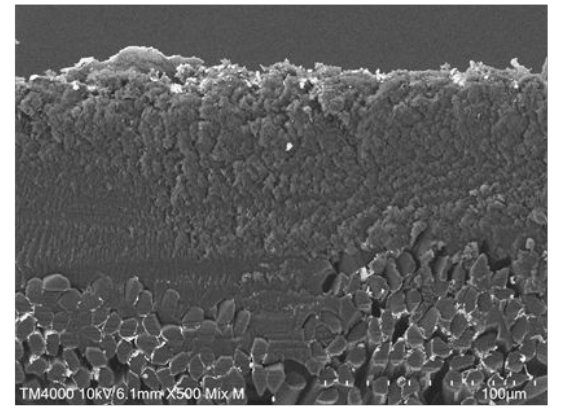

Textile 3-3

Figure S-5. Cross-sectional SEM images at 500x for film and textile capacitors (two areas per sample shown for thickness variation and textile unit structure). 
a)

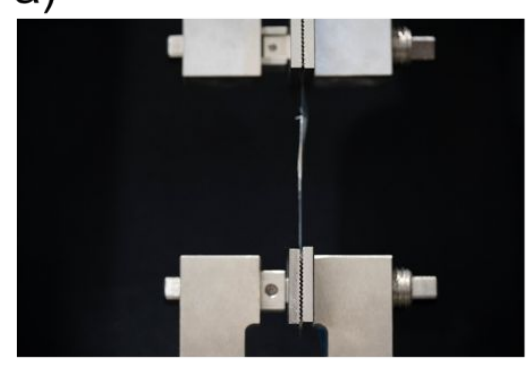

c)

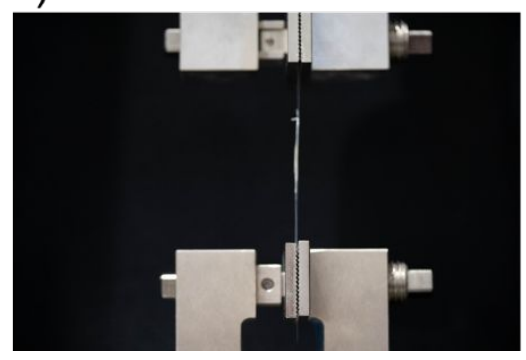

b)

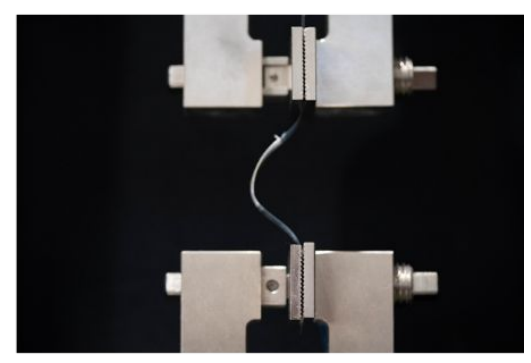

d)

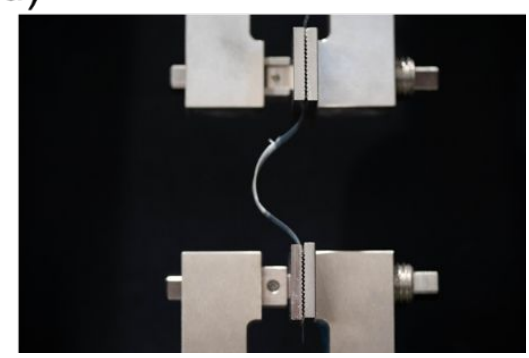

Figure S-6. Large-scale images in electromechanical performance evaluation setup in Figure 7; (a) Film 2-2 at 0\% bending strain, (b) Film 2-2 at 20\% bending strain, (c) Textile 3-1 at 0\% bending strain, and (d) Textile 3-1 at 20\% bending strain. 
a)

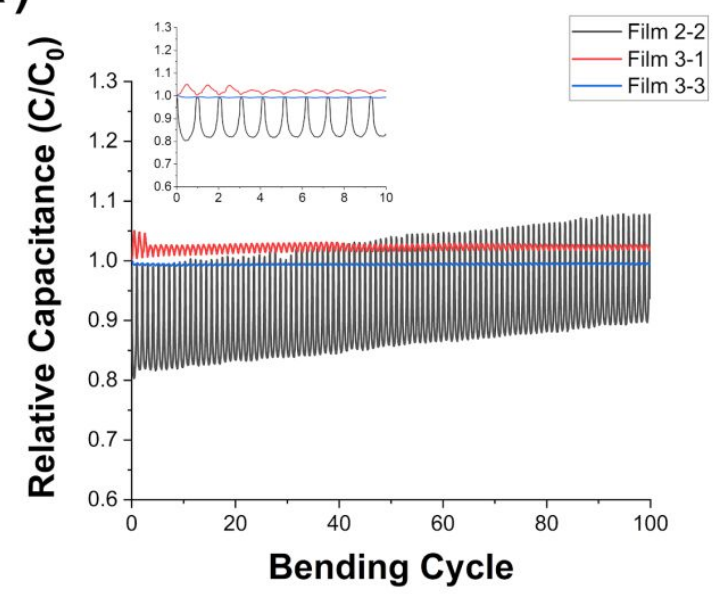

c)

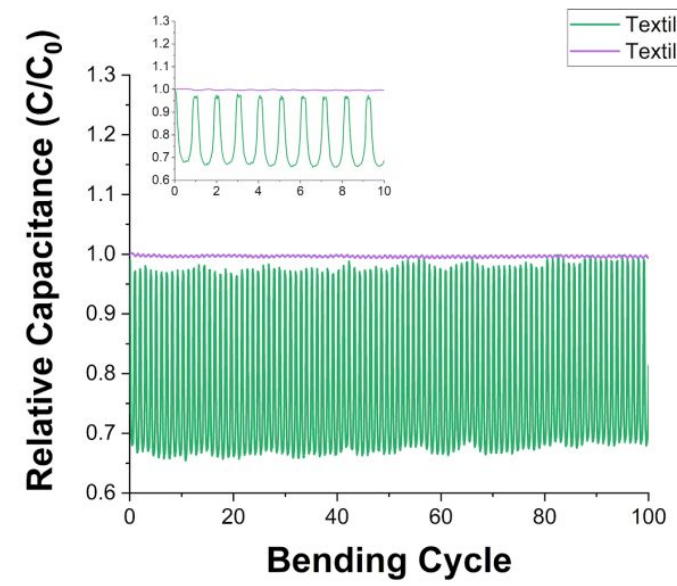

b)

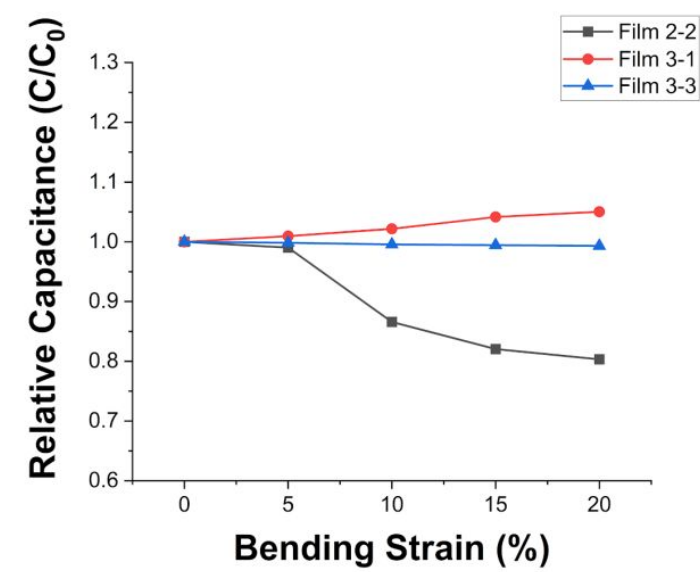

d)

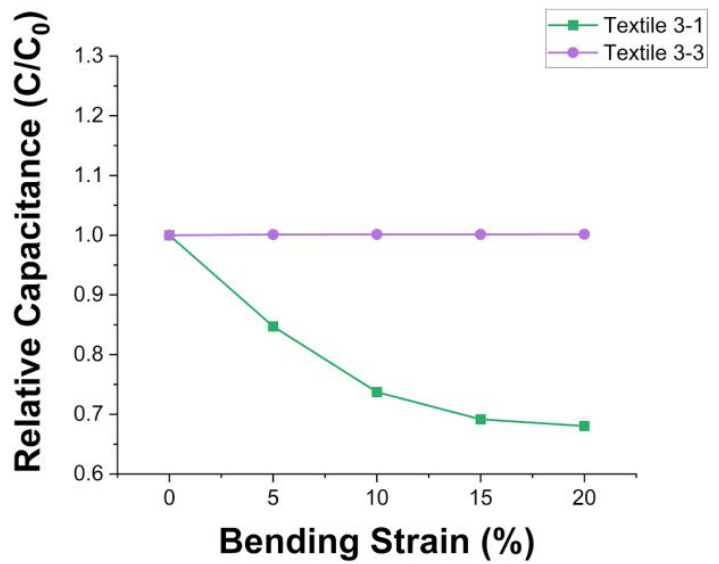

Figure S-7. Relative capacitance change in film and textile capacitors; (a) cyclic capacitance changes for the film capacitors, (b) capacitance changes for a single bending cycle at various bending strains for the film capacitors, (c) cyclic capacitance changes for the textile capacitors, and (d) capacitance changes for a single bending cycle at various bending strains for the textile capacitors. 
a)

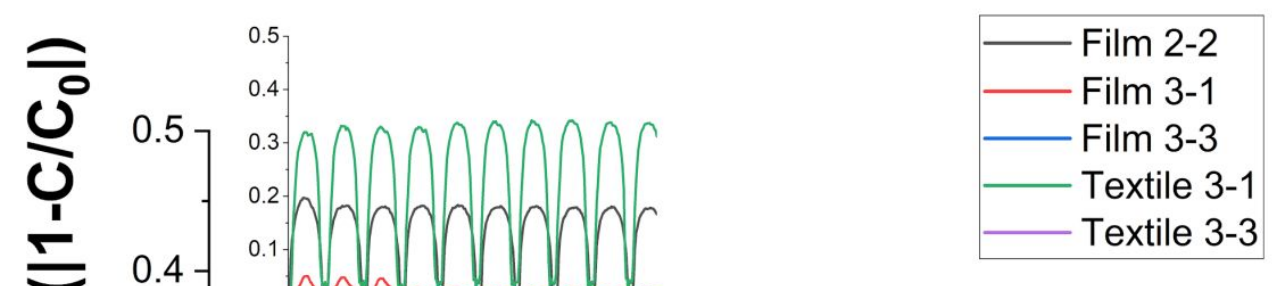

Figure S-8. Absolute capacitance change of the capacitors (combined plot). 